\title{
Accumulation Games on Graphs
}

\author{
LSE-CDAM-2008-18 \\ Steve Alpern ${ }^{1,2}$ and Robbert Fokkink ${ }^{2}$ \\ ${ }^{1}$ Mathematics Department,London School of Economics \\ Houghton Street, London WC2A 2AE, UK \\ s.alpern@lse.ac.uk \\ ${ }^{2}$ Faculty of Electrical Engineering, \\ Mathematics and Information Technology \\ Delft University, P.O.Box 5031, \\ 2600 GA Delft, Netherlands \\ R.J.Fokkink@ewi.tudelft.nl
}

Keywords: accumulation games, graphs, reliability

ORMS Classification Words: Games/group decisions, Networks/graphs MSC 2000 Classification Codes: 91A43 Games involving graphs 91A05 2-person games, 90C27 Combinatorial Optimization

September 20, 2008 


\begin{abstract}
Accumulation games on discrete locations were introduced by W. Ruckle and K. Kikuta. The Hider secretly distributes his total wealth $h \geq 1$ over locations $1,2, \ldots, n$. The Searcher confiscates the material from any $r$ of these locations. The Hider wins if the wealth remaining at the $n-r$ unsearched locations sums to at least 1; otherwise the Searcher wins. Their game models problems in which the Hider needs to have, after confiscation (or loss by natural causes), a sufficient amount of material (food, wealth, arms) to carry out some objective (survive the winter, buy a house, start an insurrection).

This paper takes the hiding locations to be the nodes of a graph and restricts the node sets the Searcher can remove to be drawn from a given family: the edges, the connected $r$-sets, or some other given sets of nodes. This models the case where the pilferer, or storm, is known to act only on a set of close locations. Unlike the original game, our game requires mixed strategies.
\end{abstract}




\section{Introduction}

Accumulation games on discrete locations were introduced by Ruckle [16] and Kikuta and Ruckle [12] to model the following problem: A Hider has an amount $h \geq 1$ of continuous material (water, food,money, arms, secrets) which he must stash secretly among $n$ locations $\mathcal{N}=\{1, \ldots, n\}$ in such a way that after any $r$ of them are searched and confiscated, the material remaining at the other locations is still enough to carry out a certain task. The amount required to be 'enough' can be normalized to 1 .

As one example of an accumulation game in real life we mention the scatter-hoarder, a term introduced by Morris [14]. This is an animal such as a squirrel who in times of surplus stashes its food in multiple caches for times when food is less plentiful. It will also scatter hoard when it can't defend a large concentration of stored food. In other words, it will spread out the food supply, perhaps throughout its home range, hiding it well to prevent other animals from finding and pilfering it. In settings such as these, it is reasonable to assume that the locations which are searched (or destroyed by Nature) cannot be arbitrary and are in some sense close; the Searcher (pilferer) may have a limited searching time, or the feared natural event may be known to destroy only locations in a limited area. See [6] and [18] for two outstanding papers in the large literature on cache-hoarding and pilfering in the animal behavior literature. Of course this ecological application is only one of many for accumulation games. Other applications are given in the original papers.

To model this extended scenario as a game, where the Searcher can only search a set of close locations, we assume that the hiding locations are the $n$ nodes of a graph $G=(\mathcal{N}, \mathcal{E})$, and that the Searcher can only search certain subsets, perhaps just edges, or maybe the connected $r$-sets. A pure strategy for the Hider remains a partitioning of the material $h$ over the nodes. However in our game the Searcher can confiscate (search) material only from a node set $S$ belonging to a given search space (or hypergraph) $\mathcal{S} \subset 2^{\mathcal{N}}$. The Hider wins (Payoff $=1)$ if the remaining material outside of $S$ sums to at least 1; otherwise the Searcher wins (Payoff=0). We call this the accumulation game $A(\mathcal{S})$. The Value $V=V(G, \mathcal{S}, h)$, which exists by standard minimax results [2], represents the probability that the Hider wins, with best play on both sides. In our notation, the original Kikuta-Ruckle game is defined by the complete graph $G=K_{n}$ on $n$ nodes, with $\mathcal{S}$ the set of all (connected) $r$-sets. 
When $\mathcal{S}=\mathcal{E}$, the edge set of a given graph, we call this the edge-accumulation game.

As an example of a game $A(\mathcal{S})$, suppose $\mathcal{S}$ is the set of $\mathcal{E}$ edges of the 'table graph' drawn below in Figure 1.

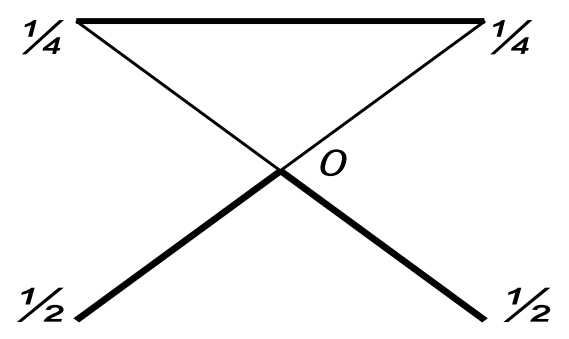

Figure 1. Table graph.

If the Hider's total wealth $h$ satisfies $h \geq 3 / 2$, he wins certainly by distributing material over the nodes as shown in the figure. Note that this distribution has the maximum total weight (namely $3 / 2$ ) such that the sum of the weights on each edge does not exceed $h-1=1 / 2$. This weighting (i) uses only 0 and two other weights with one twice the other, and (ii) respects the symmetry of the graph. These two properties of pure strategies used in optimal Hider strategies are general (Theorems 17 for $\mathrm{i}$ and Theorem 3 for ii). If $1 \leq h<3 / 2$ the Value of the game is 2/3: an optimal Searcher strategy is to choose equiprobably among the three thick edges (legs and table top) and an optimal mixed strategy for the Hider is to place all the weight at a single node, choosing the each end of the tabletop with probability $1 / 6$ and each base of a leg with probability $1 / 3$. Another approach to this game is given in Theorem 18 and Example 19.

The game $A(\mathcal{S})$ can also be interpreted as a game against Nature, played either by the Hider or the Searcher. In the former case, the Hider is a Scatter Hoarder who fears natural (or at least uncontrollable) events will destroy some of his caches, and the cache sets that may be destroyed together constitute the search space $\mathcal{S}$. In the latter case (see the game $A^{c}$ ), we consider the Searcher to be a Pilferer who knows the locations together have at least an amount $h$ of food stored in them, but not the distribution. He needs the locations $S$ that he searches to have a minimum amount $(h-1)$ for him to survive. 
The discrete location accumulation game is related (especially for the line and circle graphs) to the 'number hides game' introduced by Ruckle [15], studied by Baston, Bostock and Ferguson [4] and extended by Zoroa, Fernandez-Saez and Zoroa [19]. The main difference is the discontinuity of the payoff in our game (a game of kind), where the Searcher tries not to maximize the expected wealth found, but rather the probability of confiscating enough wealth $(h-1)$ to win the game. For a general monograph on the theory of search games, the reader is referred to [3].

The paper is organized as follows. In Section 2 we formally define the accumulation game and its complementary form, establish the existence of a Value and of optimal strategies which respect the symmetry of the search space $\mathcal{S}$. In Section 3 we solve a game on a specific graph, for all values of the wealth $h$. This section motivates the more general subsequent sections by indicating which results are needed in the specific analysis. In Section 4 we define the minimal weight problem for a graph (or hypergraph): What is the minimum total weight on the nodes of a given graph needed to make the weight of each edge (sum of weights on its ends) at least 1 ? We consider the dual problem and solve the minimum weight problem for various classes of hypergraphs. In Section 5 we show that for certain classes of graphs (e.g. bipartite graphs) our accumulation game is equivalent to the original game of Kikuta and Ruckle, described in the first paragraph of this Introduction. In Section 6 we study the accumulation game on the circle graph, where the search sets $S$ are arcs consisting of $s$ consecutive nodes. This study was begun by Kikuta and Ruckle [12], which indeed motivated our general formulation of accumulation games on graphs. They obtained bounds on the Value of this game in terms of $n, s$ and $h$; we improve their bounds. In the final Section 6 we briefly summarize the results of the paper.

\section{The Accumulation Game $A(\mathcal{S})$}

In this section we give a formal definition of the accumulation game $A(\mathcal{S})$ and discuss some of its general properties. A reader wanting to see a specific example before general material could skip ahead to Section 3 and come back to this section afterwards. 


\subsection{Formal Definition and existence of a Value}

In this section we define the game $A(\mathcal{S})$ and all of its elements. We establish the existence of a Value and optimal (mixed) strategies. We show that it can be assumed that these strategies respect the symmetry of the underlying graph, or more generally of the search space $\mathcal{S}$. We explain the role of domination arguments and define the related complementary game $A^{c}$, where the Hider wins if the set picked by the Searcher has total weight at least 1 .

Throughout the paper we use the following notation:

$n, h, r, s$ The number of hiding locations is denoted by $n$; the Hider's initial wealth is $h$, which we assume is at least 1 . When the Searcher can search a fixed number of locations, we denote this number by $r$, and the number unsearched by $s=n-r$.

$\mathcal{N}, \mathcal{N}_{r}$ The set of hiding locations is denoted by $\mathcal{N}=\{1, \ldots, n\}$ The set $\mathcal{N}$ is also the node set of any graph that we consider. $\mathcal{N}_{r}$ is the collection of all $r$-subsets of $\mathcal{N}$ (sets of cardinality $r$ ).

$G$ This is a graph with node set $\mathcal{N}$ and edge set $\mathcal{E}$.

$w$ This is a weighting $w=\left(w_{1}, \ldots, w_{n}\right)$ which hides weights $w_{i}$ at nodes $i \in \mathcal{N}$. We also use measure notation, writing $w(S)=\sum_{i \in S} w_{i}$. We require that the total weight of $w$ satisfies $w(\mathcal{N})=h$; sometimes we relax this by allowing the Hider to choose $w$ with $w(\mathcal{N}) \leq h$, since this does not help him.

$\mathcal{S}$ This is a given collection of subsets of $\mathrm{N}$, that is, $\mathcal{S} \subset 2^{\mathcal{N}}$. We call $\mathcal{S}$ the search space, and sets $S$ in $\mathcal{S}$ are called search sets, or simply searches. We also call $\mathcal{S}$ a hypergraph.

light An edge or more generally a search set $S \in \mathcal{S}$ is called light if $w(S) \leq$ $h-1$.

heavy An edge or $S \in \mathcal{S}$ is called heavy if $w(S) \geq 1$.

Definition 1 For a given search space $\mathcal{S}$, the accumulation game $A(\mathcal{S})$ is the zero-sum win-lose game in which the Hider (maximizer) picks a weighting (measure) $w$ on $\mathcal{N}$ with $w(\mathcal{N})=h$, the Searcher (minimizer) picks a search 
set $S \in \mathcal{S}$, and the Hider wins if $w(\mathcal{N}-S) \geq 1$. Setting the Payoff for a Hider win to $P=1$ and a Searcher win to $P=0$, we have

$$
P(w, S)=\left\{\begin{array}{c}
1 \text { if } w(\mathcal{N}-S) \geq 1 \\
0, \text { otherwise }
\end{array} .\right.
$$

In the complementary accumulation game $A^{c}(\mathcal{S})$ the Hider wins if $w(S) \geq 1$. Thus $A^{c}(\mathcal{S})$ is the same as the game $A(c \mathcal{S})$, where $c$ is the complement operation.

Note that the winning condition $w(\mathcal{N}-S) \geq 1$ for the accumulation game can be expressed more simply as

$$
w(S) \leq h-1 \text {, namely the Searcher picks a light set. }
$$

Although the set of weightings is infinite and $P(w, S)$ is not continuous in $w$, we can still establish the existence of a Value. Observe that if $P(w, S)<1$ then $w(\mathcal{N}-S)<1$ which remains true in an open set around $w$, so $P$ is upper semicontinuous. Since the set of weightings is compact, it follows from standard minimax theorems (e.g. [2]) that,

Theorem 2 The game $A(\mathcal{S})$ has a Value and optimal mixed strategies for both players.

In fact our analysis will show that the Hider effectively has only finitely many pure strategies so eventually this theorem will not be needed.

A mixed strategy for the Searcher is simply a distribution over the search space $\mathcal{S}$, a mixed strategy for the Hider is a finite distribution over weightings $w$, and the Value of the game is the probability that the Hider wins the game, with best play on both sides.

\subsection{Symmetry considerations}

Note that in the edge-accumulation game $(\mathcal{S}=\mathcal{E})$ on the table graph drawn in Figure 1, the players had optimal strategies that respected the symmetry of the graph. We now show that this is always possible. In the following, the reader is advised to think of the search space $\mathcal{S}$ as the set of edges of a given graph. However our definitions will hold when $\mathcal{S}$ is any family of subsets of $\mathcal{N}$ (formally, a hypergraph on $\mathcal{N}$ ). Given $\mathcal{S}$, we say that a bijection 
$\gamma: \mathcal{N} \rightarrow \mathcal{N}$ is an automorphism (of $(\mathcal{N}, \mathcal{S})$ ) if $S$ belongs to $\mathcal{S}$ if and only if $\gamma(S)$ belongs to $\mathcal{S}$. We denote the group of all automorphisms (sometimes called symmetries) by $\Gamma$. For example if $\mathcal{S}$ is the edge set of the graph of Figure 1, there are four automorphisms $t_{1}^{i} t_{2}^{j}, i, j=0,1$, with $t_{1}$ denoting the transposition of the two top nodes and $t_{2}$ the transposition of the two bottom nodes. Let $A^{\prime}(\mathcal{S})$ denote the symmetrized version of the game in which after the players choose $w$ and $S$, a random (equiprobable) element $\gamma$ is chosen from $\Gamma$ and the payoff is $P(\gamma w, S)=P\left(w, \gamma^{-1} S\right)$. Observe that either player can ensure that the symmetrized version of the game is played, simply by applying a random automorphism to their own strategy. Since either player can ensure that $A^{\prime}$ is played, its value must be the same as that of the original game $A$. This symmetry argument is not new, see [1] for a more formal group-theoretic proof (in a related context). Summarizing, we have the following result on symmetry.

Theorem 3 In the game $A(\mathcal{S})$, both players have optimal strategies which respect (are invariant under) the symmetry of $\mathcal{S}$. In particular, we may view mixed strategies of the Searcher as distributions over the equivalence classes of $\mathcal{S}$, where $S_{1}$ and $S_{2}$ are equivalent if $S_{2}=\gamma S_{1}$ for some $\gamma \in \Gamma$.

To illustrate the last sentence, observe that the optimal strategy for the Searcher in the graph of Figure 1 when $h<3 / 2$ was the distribution $(2 / 3,0,1 / 3)$ over the three edge equivalence classes: legs, supports, tabletop. Once the class is chosen, the individual edge (or search) is chosen equiprobably.

A particular case of Theorem 3 occurs when all the search sets in $\mathcal{S}$ are equivalent, that is, when there is a single equivalence class. We call $\mathcal{S}$ transitive in this case. (If $\mathcal{S}=\mathcal{E}$, the graph is called edge-transitive). Since the Searcher has a single pure strategy available (pure in the symmetrized game), of picking a random $S$ in $\mathcal{S}$, his behavior is known to the Hider. Consequently the game $A(\mathcal{S})$ for transitive $\mathcal{S}$ is really an optimization problem (rather than a game) for the Hider: find the weighting $w$ satisfying $w(\mathcal{N}-S) \geq 1$ (or equivalently $w(S) \leq h-1$ ) for the maximum number of sets $S \in \mathcal{S}$, and randomly assign the weights $w_{i}$ to the nodes. That is, the Hider aims to maximize the number of light edges (or search sets $S$ ). In the simpler complementary version, he seeks to maximize the number of heavy search sets. 
Theorem 4 For the game $A(\mathcal{S})$ with $\mathcal{S}$ is transitive, the Searcher simply picks a random search set $S$ and the Hider solves the optimization problem:

$$
\max _{w}|\{S \in \mathcal{S}: w(\mathcal{N}-S) \geq 1\}| .
$$

For the complementary game $A^{c}(\mathcal{S})$, he solves the problem

$$
\max _{w}|\{S \in \mathcal{S}: w(S) \geq 1\}| .
$$

A particular example of a transitive $\mathcal{S}$ occurs in the following.

Definition 5 Ruckle's game $R(n, r, h)$ is the accumulation game $A(\mathcal{S})$ where $\mathcal{S}=\mathcal{N}_{r}$ consists of all r-subsets of $\mathcal{N}$. We denote the Value of this game by $\operatorname{VR}(n, r, h)$.

Ruckle's game, (or the Kikuta-Ruckle Game, see [16], [12]) can be viewed in our terms as taking $\mathcal{S}$ to be the connected $r$-sets on the complete graph. These games are important in themselves and also important because other games are equivalent to Ruckle games (see Section 5). An important conjecture of Kikuta and Ruckle is the following (they made it for complete graphs only)

Conjecture 6 (Generalized Ruckle) For any transitive search space $\mathcal{S}$, there is an optimal Hider strategy $w$ for $A(\mathcal{S})$ with all its nonzero weights equal.

Kikuta and Ruckle [12] established the following positive instance of the Conjecture 6 for the Ruckle game $R(n, r, h)$ : The conjecture holds if $r=$ $n-1$, when the Hider divides $h$ into $\lfloor h\rfloor$ equal nonzero weights It also holds if $r=1$, where the Hider either divides $h$ into $n$ equal weights uniformly over all locations, if $n(h-1) \geqq h$ and else he puts one weight $h$. The authors, together with K. Kikuta, have established further instances, for a paper in preparation.

\subsection{Domination and equivalence of Hider strategies $w$}

It is common in game theory to use the notion of dominance to reduce the number of strategies that need to be considered. We say that one pure strategy dominates another of the same player if it does as least as well 
against any opponent strategy. If they do the same against any opponent strategy we will say they are strategically equivalent. For a fixed search space $\mathcal{S}$ we define for any weighting $w$ the winning set (sets $S$ it wins against) $\mathcal{S}_{w}=\{S \in \mathcal{P}(w, S)=1\}$. In the accumulation game the winning sets are the light sets, those with $w(S) \leq h-1$; in the complementary game they are the heavy sets with $w(S) \geq 1$. Clearly $w^{\prime}$ dominates $w$ iff $\mathcal{S}_{w^{\prime}} \supset \mathcal{S}_{w}$ and $w^{\prime}$ is strategically equivalent to $w$ iff $\mathcal{S}_{w^{\prime}}=\mathcal{S}_{w}$. Since there are only finitely many possible sets $\mathcal{S}_{w}$, the Hider can restrict himself to a fixed strategy for each of these (or even to each of the maximal sets $\mathcal{S}_{w}$ ), perhaps the one with minimal $w(\mathcal{N})$, essentially giving him a finite pure strategy space.

\section{An Example of an Edge Accumulation Game}

To illustrate some of the ideas in the previous section and to motivate some of the results in the next section, we now give a complete analysis of the complementary edge-accumulation game on graph drawn below in Figure 2. Some of our observations will not be rigorously established until later, so to some extent this section is out of place logically - but we feel this will be compensated by giving the reader a stronger intuition about the game. Some observations made in this section may not be fully understood until the reader comes back here after completing the paper.

For this graph $n=6$ and $\mathcal{S}$ is the set $\mathcal{E}$ of edges. There are two nontrivial symmetries of the graph: the reflections with respect to the horizontal axis on the left and on the right. There are four equivalence classes of edges, as indicated in the figure. In this game, the Hider picks a weighting $w$ over the six nodes with total weight $w(\mathcal{N}) \leq h$, the Searcher picks an edge equivalence class $e$, and the Hider wins if $w(e) \geq 1$. A preliminary observation is that the weight on the terminal node of $e_{1}$ can be moved to the right end of $e_{1}$ without losing any heavy edges. Consequently $e_{1}$ dominates $e_{2}$ (this can also be seen by comparing columns $e_{1}$ and $e_{2}$ of table 1 below), so $e_{2}$ will be ignored in 
the strategic analysis.

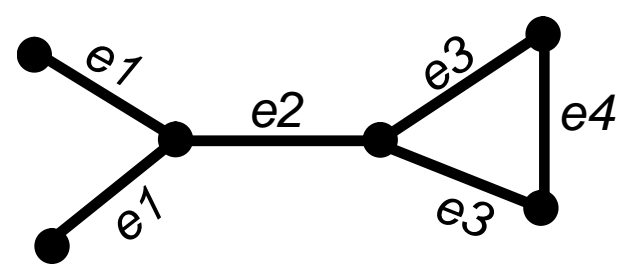

Figure 2. A graph G.

Since two weightings are equivalent if they produce the same heavy subgraph, the Hider must see which subgraphs he can make heavy, for any particular $h$. It turns out (see Theorem 15) that the Hider need only uses weights $0,1 / 2$ and 1 , so we need only consider $h$ of the form $k / 2$ for integer $k$. The table below lists all the strategies in the symmetrized game, listing all the maximal heavy subgraphs obtainable for $h=1,3 / 2,2,5 / 2$. For each of these values of $h$, we list all the subgraphs of $G$ which the Hider can make heavy, and indicate the weighting of the minimal sum which makes it heavy. For the time being, the reader may ignore the (blue) edge weights which are indicated on each graph. (Theorem 8 shows these edge weights prove that the node weight $w$ is indeed the one with minimal total weight which makes the graph heavy.) 


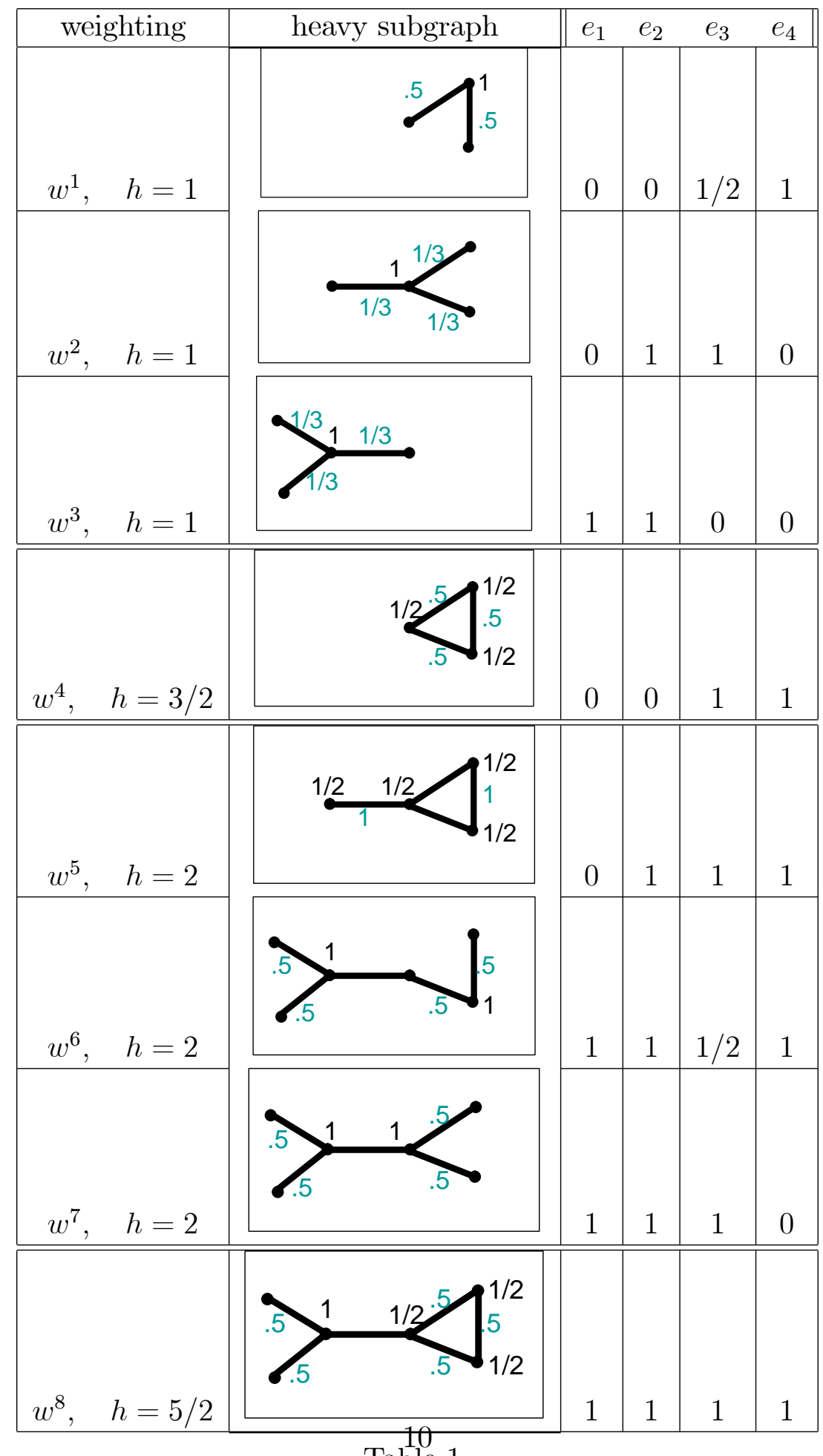

Table 1 
If $h=1$ we can assume all the weight is placed at a single node, as the alternative of $1 / 2$ at both ends of an edge is dominated by this. The three non-equivalent nodes yield the three depicted subgraphs. Note that these three graphs are all edge transitive and so the indicated edge weights and the weightings with 0's and 1's are a consequence of Theorem 10. Hence the matrix game corresponding to $h=1$ (and hence for $1 \leq h<3 / 2$ ) is (omitting the dominated column $e_{2}$ ).

\begin{tabular}{|c||c|c|c|}
\hline \multicolumn{1}{|c||}{} & $e_{1}$ & $e_{3}$ & $e_{4}$ \\
\hline$w^{1}$ & 0 & $1 / 2$ & 1 \\
\hline$w^{2}$ & 0 & 1 & 0 \\
\hline$w^{3}$ & 1 & 0 & 0 \\
\hline
\end{tabular}

The Value is $2 / 5$ and the optimal mixed strategies are $(2 / 5,1 / 5,2 / 5)$ for the Hider (rows) and $(2 / 5,0,2 / 5,1 / 5)$ for the Searcher (columns)

If the Hider's wealth goes up to $h=2$, he has three new strategies $w^{5}, w^{6}$ and $w^{7}$, which dominate the previous four strategies and lead to the matrix game

\begin{tabular}{|c||cc|c|}
\hline & $e_{1}$ & $e_{3}$ & $e_{4}$ \\
\hline$w^{5}$ & 0 & 1 & 1 \\
\hline$w^{6}$ & 1 & $1 / 2$ & 1 \\
\hline$w^{7}$ & 1 & 1 & 0 \\
\hline
\end{tabular}

The strategy $e_{2}$ is of no use to the Hider as it is dominated by all the other strategies. Here there is a symmetry $w^{5} \longleftrightarrow w^{7}, e_{1} \longleftrightarrow e_{4}$, so we can assume $w^{5}$ and $w^{7}$ are played with the same probability and $e_{1}$ and $e_{4}$ are played with the same probability, giving

\begin{tabular}{|c|c|c|}
\hline \multicolumn{2}{|r|}{$e_{3}$} & $e_{1}+\frac{1}{2} e_{4}$ \\
\hline$\frac{1}{2} w^{5}+\frac{1}{2} w^{7}$ & 1 & $1 / 2$ \\
\hline$w^{6}$ & $1 / 2$ & 1 \\
\hline
\end{tabular}

The solution is for each row and column to be played with probability $1 / 2$, giving a Value of $3 / 4$ for all $h$ with $2 \leq h<5 / 2$. That is, play $\left(w^{6}, w^{5}, w^{7}\right)$ and $\left(e_{3}, e_{1}, e_{4}\right)$ both with probabilities $(1 / 2,1 / 4,1 / 4)$. If $h \geq 5 / 2$ the Hider can force a win (Value $=1$ ) with the weighting $w^{7}$. 


\section{The Minimal Weight Problem}

In this section we justify some of the claims made in the analysis used for the graph $G$ of the previous section. In Table 1 we claimed that the weighting $w$ associated with each subgraph was the one with minimal total weight which made every edge $e$ of the subgraph heavy $(w(e) \geq 1)$. For the graphs associated to $h=1$ this is obvious, as if $h<1$ obviously no edge can be heavy. So we take as our example the graph associated with $h^{5}$.

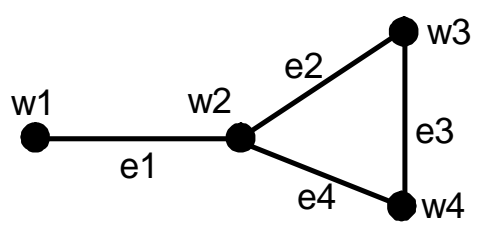

Figure 3. Graph for $h^{5}$

\subsection{Linear programming formulation}

The linear program for the minimal weight problem on this graph is

$$
\begin{array}{cc}
\operatorname{minimize} w_{1}+w_{2}+w_{3}+w_{4}, \text { subject to } \\
w_{1}+w_{2} \geq 1 \quad\left(e_{1} \text { heavy }\right) \\
w_{2}+w_{3} \geq 1 \quad\left(e_{2} \text { heavy }\right) \\
w_{3}+w_{4} \geq 1 \quad\left(e_{3} \text { heavy }\right) \\
w_{4}+w_{5} \geq 1 \quad\left(e_{4} \text { heavy }\right) \\
\text { all } w_{i} \geq 0
\end{array}
$$

The dual maximum problem, when we call the dual variables $y_{j}$, is

$$
\begin{gathered}
\text { maximize } y_{1}+y_{2}+y_{3}+y_{4} \text { subject to } \\
\leq 1(\text { edges at node } 1) \\
y_{1} \leq 1(\text { edges at node } 2) \\
\left.y_{1}+y_{2} \leq 1 \text { (edges at node } 3\right) \\
y_{3}+y_{4} \leq 1(\text { edges at node } 4) \\
y_{2}+y_{4} \leq 1 \text { all } y_{j} \geq 0 .
\end{gathered}
$$


This is the maximal edge weight problem, maximize the sum of the edge weights subject to the condition that the sum of the weights of the edges incident at a node are at most 1 . The optimal solution pair, as indicated in Table 1 , is $w_{1}=w_{2}=w_{3}=w_{4}=1 / 2$ and $e_{1}=e_{3}=1, e_{2}=e_{4}=0$, with common value $h=2$.

Definition 7 Let a search space (hypergraph) $\mathcal{S}$ on $\mathcal{N}$ be given. Then a weighting $w$ (on the nodes) is called heavy if $w(S) \geq 1$ for every search set $S \in \mathcal{S}$ and a measure $y$ on $\mathcal{S}$ is called a light weight if for every $i \in \mathcal{N}$ we have $y\left(\mathcal{S}_{i}\right) \leq 1$. Here $\mathcal{S}_{i}=\{S \in \mathcal{S}: i \in S\}$ denotes the collection of all search sets containing $i$. A heavy weight $w$ minimizing the total weight $w(\mathcal{N})$ is called a minimal heavy weight; a light weight $y$ maximizing the total weight $y(\mathcal{S})$ is called a maximal light weight.

This duality holds more generally for arbitrary search families (hypergraphs) $\mathcal{S}$, not just edges of a graph. Then the weak duality theorem gives the following result, which justifies the claims made about the minimality of the weightings in Table 1 as a consequence of the blue (light colored) numbers on the edges.

Theorem 8 Let a search family $\mathcal{S}$ be given. If a heavy weight $w$ on $\mathcal{N}$ and a light weight $y$ on $\mathcal{S}$ have the same total weight $h=w(\mathcal{N})=y(\mathcal{S}) \equiv$ $\sum_{S \in \mathcal{S}} y(S)$ then $w$ is a minimal heavy weight and $y$ is a maximal light weight.

\subsection{Transitive hypergraphs $\mathcal{S}$}

If the search space $\mathcal{S}$ is transitive, then the solution to the minimal node weight and maximal edge (or $\mathcal{S}$ ) weight problem is easy. The solution for an edge-transitive graph is shown in Figure 4 below, where all the edges $(S$ in $\mathcal{S}=\mathcal{E}$ ) have equal weight and the nodes of maximal degree have weight 1 with other nodes having weight zero. 


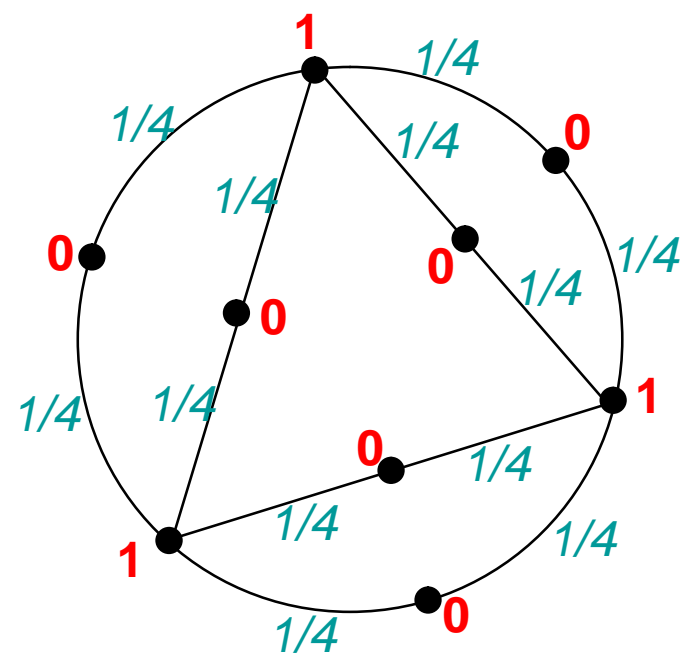

Figure 4. Optimal node and edge weights

The situation is Figure 4 holds for all edge-transitive graphs, and more generally for all transitive hypergraphs $\mathcal{S}$.

Lemma 9 If $\mathcal{S}$ is transitive, then there is a uniform distribution $(y(S)$ constant) which is a maximal light weight on $\mathcal{S}$.

Proof. If $y$ is a light-weight on $\mathcal{S}$ and if $\alpha$ is an automorphism of $(\mathcal{N}, \mathcal{S})$, then $\alpha(\mathbf{y})$ is again light and has the same total weight. The average of lightweights is again a light weight. So if $y$ is any minimal light weight, then the average $u$ over all automorphims $\alpha(\mathbf{y})$ is also a minimal light-weight and it is uniform.

Since we can solve the dual problem, we can also solve the primal. The following result settles the generalized Ruckle conjecture for the case that the payoff is 1 , that is, when the Hider can ensure a win (Value is 1). An example is given in Figure 4, where the minimal weighting puts 1 on the three nodes of maximal degree 4 and 0 on the other nodes. 
Theorem 10 If $\mathcal{S}$ is transitive, then there is a minimal heavy weight $w$ which is constant on the nodes of maximal degree (the $i$ which belong to the greatest number of sets $S$ in $\mathcal{S}$ ) and zero elsewhere.

Proof. Let $m$ be the cardinality of $\mathcal{S}$ and let $d$ be the maximal degree (maximal number of $S \in \mathcal{S}$ containing a common node). By the previous lemma there is a maximal light weight $y$ with $y(S)=c$, a constant. So at a node of maximal degree the lightness condition gives $c \cdot d \leq 1$ and the maximal condition then gives $c=1 / d$. Hence the total weight $y(\mathcal{S})$ is $m / d$ and consequently by Theorem 8 any heavy weight $w$ with $w(\mathcal{N})=m / d$ must be minimal. We will define such a $w$ which has the properties stated in the theorem.

Since $\mathcal{S}$ is transitive, each set $S \in \mathcal{S}$ contains the same number $k$ of nodes of maximal degree. Let us count the nodes of maximal degree. Each $S \in \mathcal{S}$ has $k$ of them and there are $m$ different sets $S$, so that gives us $k m$, but each node of maximal degree is counted for $d$ distinct $S$. So in all there are $\mathrm{km} / \mathrm{d}$ nodes of maximal degree. Let $w$ be the weighting that puts $1 / k$ on these nodes and 0 elsewhere. Then $w(S)=k(1 / k)=1$ for every $S \in \mathcal{S}$, so $w$ is a heavy weight. But the total weight of $w$ is $(k m / d)(1 / k)=m / d$, so as observed in the previous paragraph it must be a minimal heavy weight.

Note that the $w$ of the Theorem has only one non-zero weight namely $1 / k$. This is the property in Conjecture 6 . Note how the past two results apply to the transitive winning graphs of weightings $w^{1}$ to $w^{4}$ in Table 1 . An immediate consequence of Theorem 10 is the following.

Corollary 11 Given a transitive hypergraph $\mathcal{S}$, suppose $h$ is sufficiently large so that the Hider can win the accumulation game $A(\mathcal{S})$, that is, the Value is 1. Then the generalized Ruckle conjecture holds.

A graph is called regular of degree $d$ if there are exactly $d$ edges incident to every node. For such graphs we have the following.

Theorem 12 If $G$ is a regular graph of degree $d$, then the minimal weight problem (with $\mathcal{S}=\mathcal{E}$ ) is solved by putting weight $1 / 2$ at all nodes.

Proof. The stated weight has $w(\mathcal{N})=n / 2$. Consider the feasible solution to the dual edge weight problem which puts $1 / d$ on every edge. The sum of the edge weights is $m / d$, where $m$ is the number of edges. Thus we have $m / d \leq n / 2$ by duality. But if $G$ is regular, the number of nodes is given by $m=n d / 2$. Consequently $m / d=n / 2$ and the two solutions given to the dual problems are both optimal. 


\subsection{Minimal heavy weightings on graphs}

The reader will have noticed that all the minimal weights $w^{i}$ in the example of Section 3, those listed in Table 1 , use only the values $0,1 / 2$ and 1 . This property holds for all graphs, as we establish in Theorem 14 . Of course there may be other minimal weights, for example putting $1 / 3$ and $2 / 3$ on the two nodes of a graph consisting of a single edge. For general hypergraphs, this property obviously does not hold. Consider, for example, the case where $\mathcal{S}$ consists of all four 3 -sets of $\mathcal{N}=\{1,2,3,4\}$ and $h=4 / 3$. Here, using only 0 , $1 / 2$ and 1 we can make at most three sets heavy (putting 1 on some node), while putting $1 / 3$ on every node makes all four sets heavy and is a minimal heavy weighting.

Call a weighting $w$ nontrivial if $0<w_{i}<1$ for some $i \in \mathcal{N}$. For nontrivial weightings $w$, define $b=b(w)=\max \left\{w_{i}: w_{i} \neq 1\right\}$ and $a=$ $\min \left\{w_{i}: w_{i} \neq 0\right\}$, and call the $w_{i}$ equal to $b$ and $a$ respectively, the $b i g$ weights and the small weights.

Lemma 13 Let $\mathcal{S}=\mathcal{E}$ be the edge set of a graph on nodes $\mathcal{N}$, and let $w$ be any nontrivial minimal heavy weighting. Then $a(w)+b(w)=1$ and the respective numbers $\beta$ and $\alpha$ of big and small weights are equal.

Proof. We cannot have $a+b>1$ because then $w(\mathcal{N})$ would be smaller if all the big weights were reduced to $1-a$. We cannot have $a+b<1$ because then $w(\mathcal{N})$ would be smaller if we reduced all the small weights to zero. Hence $a+b=1$. If $a=b$, we are done. So suppose that $a<b$. Let $\varepsilon>0$ be the minimum difference between any two weights $w_{i}$. We cannot have $\beta>\alpha$ because then we could reduce $w(\mathcal{N})$ by changing the big weights to $b-\varepsilon$ and increasing the small weights to $a+\varepsilon$. Similarly we cannot have $\alpha>\beta$ because then we could reduce $w(\mathcal{N})$ by decreasing the small weights and increasing the big weights. Hence $\alpha=\beta$.

Theorem 14 Let $\mathcal{S}=\mathcal{E}$ be the edge set of a graph on nodes $\mathcal{N}$. Then there is a minimal heavy weighting $w$ with all $w_{i} \in\{0,1 / 2,1\}$.

Proof. Let $M$ denote the nonempty compact convex subset of minimal heavy weightings. If $M$ contains a trivial weighting with all $w_{i} \in\{0,1\}$, we are done. Otherwise, the difference function $\delta(w)=b(w)-a(w)$ is upper semicontinuous and is minimized at some nontrivial minimal heavy weighting we will just call $w$. By Lemma 13, we have $a+b=1$ and $\alpha=\beta$. If $a<b$ we 
can reduce $\delta$ by making all the big weights smaller, and all the small weights bigger, by the same small amount. So $a=b$ and since $a=b=1$ we have $a=b=1 / 2$. Consequently we have $w_{i} \in\{0,1 / 2,1\}$ for all $i \in \mathcal{N}$.

The significance of this result for complementary accumulation games, like the one analyzed in Section 3, is that every weighting is dominated by a minimal heavy weighting with all of its weights either $0,1 / 2$ or 1 . Consequently the Hider can restrict to using mixed strategies concentrated on such pure strategies, as we demonstrated for a particular example in Section 3. It follows that new strategies for the Hider (and hence new Values for the game) appear only when $h=K / 2$ for some integer $K$. Summarizing these observations, we have the following Corollary of Theorem 14.

Theorem 15 For the complementary accumulation game $A^{c}(\mathcal{E})$, where $\mathcal{E}$ is the edge set of any graph $G$, there exists an optimal mixed strategy for the Hider that is concentrated on pure strategies (weightings) that have all their weights in in the set $\left\{0, \frac{1}{2}, 1\right\}$. Consequently the Value function for this game is constant for $h \in\left[\frac{K-1}{2}, \frac{K}{2}\right)$, for all integers $K$.

For the original edge accumulation game, the idea is the same, but it is a bit trickier. Here, the Hider wins if the edge $e$ picked by the Searcher satisfies $w(\mathcal{N}-e) \geq 1$, or simply $w(e) \leq h-1 \equiv \gamma$. Call an edge $e$ light, or $\gamma$-light, if $w(e) \leq \gamma$. A weighting is light if it makes every edge light. Scaling Theorem 14 is terms of $\gamma$-heavy (with weights $w_{i}$ in $\{0, \gamma / 2, \gamma\}$ ) and then using the reflection $x \rightarrow \gamma-x$, we obtain the following equivalent result.

Corollary 16 Let $\mathcal{S}=\mathcal{E}$ be the edge set of a graph on nodes $\mathcal{N}$, with every node in some edge, and let $\gamma>0$ be given. Then there is a maximal $\gamma$-light weighting $w$ with all $w_{i} \in\{0, \gamma / 2, \gamma\}$.

We can now obtain the analogous result to Theorem 14 for edge accumulation games.

Theorem 17 In the edge accumulation game $A(\mathcal{E})$, every strategy (weighting) $\bar{w}$ is dominated by a weighting $w$ with $w(\mathcal{N})=h$ and either all the weight $h$ is placed at a single node $i$ with $w(i)=h$, or there are at most two non-zero weights, with one twice the other. Consequently there is an optimal Hider mixed strategy which uses only pure strategies (weightings) with these properties. 
Proof. Let $\gamma=h-1$ and let $\mathcal{E}^{\prime} \subset \mathcal{E}$ denote the $\gamma$-light edges of $\bar{w}$. If $\bar{w}_{i}>\gamma$ for some node $i$, then setting $w_{i}=h$ and $w_{j}=0$ otherwise gives a dominating strategy satisfying (i). If not, let $w^{\prime}$ be the maximal light weighting given by Corollary 16 with $\mathcal{S}=\mathcal{E}^{\prime}$. In particular, every edge $e$ which is light for $\bar{w}$ is light for $w^{\prime}$. Since $w^{\prime}$ is maximal, this means $w^{\prime}(\mathcal{N}) \geq \bar{w}(\mathcal{N})=h$, as $\bar{w}$ is a light weight on $\mathcal{E}^{\prime}$ (by definition of $\mathcal{E}^{\prime}$ ). Finally, define $w$ by the scaling $w=w^{\prime}\left(h / w^{\prime}(\mathcal{N})\right) w^{\prime}$, so that $w(\mathcal{N})=h$ and $w$ is a feasible strategy.
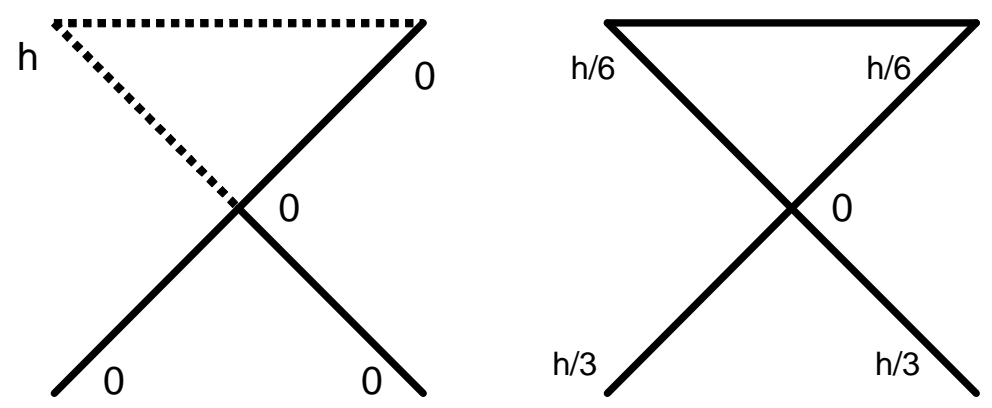

Figure 5. Optimal weightings with indicated solid winning sets.

The above results for edge accumulation games can be illustrated by the earlier discussion of the table graph of Figure 1, in the Introduction. For $h=3 / 2$ there is a type (ii) weighting with weights $0,1 / 4$ and $1 / 2$, and for $h=1$ there is a type (i) weighting with all the weight at a leg bottom. More generally, Figure 5 shows a type (i) strategy for $1 \leq h<3 / 2$ on the left and a type (ii) strategy for $h \geq 3 / 2$. Of course we could equally well replace the $h$ on the left by a 1 , if we are not required to use all the available wealth (if we can have $w(\mathcal{N})<h$ ). The situation on the left will hold whenever the winning set of edges (solid lines, in the figure) does not contain all the nodes. In the edge accumulation game, any such set of edges can be made winning (light) by putting all the weight on a node they do not contain.

\section{Games equivalent to Ruckle's Game}

In this section we demonstrate that some accumulation games on graphs are equivalent to Ruckle's game $R(n, r, h)$ with $r=1$ or $r=n-1$, as 
defined in Definition 5. The solution obtained by Kikuta and Ruckle for these parameter values are described at the end of Section 2.2.

Consider for example the Table Graph of Figure 1. Suppose the Hider restricts himself to weightings $w_{1}, w_{2}, w_{3}$ on the following three nodes: left table top and left and right leg bottoms. Similarly, the Searcher restricts himself to the following three edges: the two legs and the table top. Then they are effectively playing Ruckle's game with $n=3, r=1$, and whatever $h$ they were playing with on the table graph. The solution to Ruckle's game here is $w_{1}=w_{2}=w_{3}=1 / 2$ if $h \geq 3 / 2$ and to place a unit weight randomly on one of the three locations otherwise. This is a different strategy than given in the Introduction, but gives the same value. In this section we will show why this analysis works for a class of graphs. Thus the accumulation game of the Table Graph is equivalent to $R(3,1, h)$.

To make this argument more general, we extend some observations for the circle and line graphs that were made in [12]. For any search family $\mathcal{S}$ on $\mathcal{N}$, call a subset $B$ of $\mathcal{N}$ an independent set if no search $S$ in $\mathcal{S}$ contains two elements of $B$, and call a subset $\mathcal{T}$ of $\mathcal{S}$ a covering family if the union of its sets is $\mathcal{N}$. Define Ind $(\mathcal{S})$, the node independence number, to be the maximum size of an independent set, and $\operatorname{Cov}(\mathcal{S})$, the covering number, to be the minimum size of a covering family. Since every set $S$ in a covering family can contain at most one node of an independent set, we always have $\operatorname{Ind}(\mathcal{S}) \leq \operatorname{Cov}(\mathcal{S})$. For example the edge family of the table graph of Figure 1 has Ind $=\operatorname{Cov}=3$, and the bowtie graph of Figure 2 has Ind $=2$ and $\operatorname{Cov}=3$. If $\mathcal{S}$ denotes the connected $r$-sets of the line graph we have $\operatorname{Ind}(\mathcal{S})=\operatorname{Cov}(\mathcal{S})=\left\lceil\frac{n}{r}\right\rceil$. For the following recall that $V R(n, r, h)$ is the Value of Ruckle's game.

Theorem 18 For any search family $\mathcal{S}$ on $\mathcal{N}$, the value $V(\mathcal{S})$ of the accumulation game $A(\mathcal{S})$ satisfies the inequalities

$$
V R(\operatorname{Ind}(\mathcal{S}), 1, h) \leq V(\mathcal{S}) \leq V R(\operatorname{Cov}(\mathcal{S}), 1, h)
$$

If $\operatorname{Ind}(\mathcal{S})=\operatorname{Cov}(\mathcal{S})=u$, then the game $A(\mathcal{S})$ is equivalent to Ruckle's accumulation $R(u, 1, h)$.

Proof. To obtain the left hand inequality, let $u=\operatorname{Ind}(\mathcal{S})$ and let $\left(w_{1}, \ldots, w_{u}\right)$ be an optimal weighting for Ruckle's game $R(u, 1, h)$. If the Hider places these weights randomly on some maximal independent set for $\mathcal{S}$, then any 
Search strategy $S \in \mathcal{S}$ can contain only one of these, by definition of independent set. Hence his expected payoff is at least $V R(u, 1, h)$.

To obtain the right hand inequality, consider the Searcher strategy of choosing equiprobably among the $\operatorname{Cov}(v)$ searches $S$ in a fixed minimal covering set of $\mathcal{S}$. Thus every weight is found with probability at least $1 / \operatorname{Cov}(\mathcal{S})$, that is, with at least the same probability as in Ruckle's game. This gives the right inequality.

Example 19 Let $\mathcal{S}$ denote the edges of the Table Graph of Figure 1. A maximal independent set consists, for instance, of the two leg bottoms and the left end of the table top. A minimal covering edge set consists of the two legs and the table top. So Ind $(\mathcal{S})=\operatorname{Cov}(\mathcal{S})=3$. The optimal strategies given in the Introduction are the 'unsymmetrized' versions of the strategies in Ruckle's game with one search location.

We say that a graph is a Ruckle graph if $\operatorname{Ind}(\mathcal{S})=\operatorname{Cov}(\mathcal{S})$, so the table graph is a Ruckle graph and so are all bipartite graphs by an old result of Konig [13].

For any search family $\mathcal{S}$ on $\mathcal{N}$, call a subfamily $\mathcal{T}$ of $\mathcal{S}$ an independent family if the searches (sets) in $\mathcal{T}$ are pairwise disjoint, and call a subset $B$ of $\mathcal{N}$ a node cover if each search $S$ in $\mathcal{S}$ contains an element of $B$. Define $C o-\operatorname{Ind}(\mathcal{S})$, the co-independence number, to be the maximum size of an independent family, and $\operatorname{Co}-\operatorname{Cov}(\mathcal{S})$, the co-covering number, to be the minimum size of a covering set.

Theorem 20 For any search family $\mathcal{S}$, the value $V^{c}(\mathcal{S})$ of the complementary accumulation game $A^{c}(\mathcal{S})$ satisfies the inequalities

$V R(\operatorname{Co}-\operatorname{Cov}(\mathcal{S}), \operatorname{Co}-\operatorname{Cov}(\mathcal{S})-1, h) \leq V^{c}(\mathcal{S}) \leq V R(\operatorname{Co}-\operatorname{Ind}(\mathcal{S}), \operatorname{Co}-\operatorname{Ind}(\mathcal{S})-1, h)$

Hence if $C o-\operatorname{Ind}(\mathcal{S})=C o-\operatorname{Cov}(\mathcal{S})=u$ then the complementary game is equivalent to Ruckle's accumulation game $R(u, u-1, h)$.

Proof. First note that for any $u$, the value $V R(u, u-1, h)$ is equal to the value of the complementary accumulation game on the complete graph, the value $V R^{c}(u, 1, h)$ of the complementary Ruckle game where the Searcher picks a single random node and the Hider wins if its weight is at least 1. 
To prove the left hand inequality, set $u=\operatorname{Co}-\operatorname{Cov}(\mathcal{S})$ and let $\left(w_{1}, \ldots, w_{u}\right)$ be an optimal weighting for $R^{c}(u, 1, h)$. Consider the Hider strategy in $B^{c}(\mathcal{S})$ which puts these weights randomly on the $u$ nodes of some fixed minimal covering set $B$ of $\mathcal{S}$. Each search set $S$ in $\mathcal{S}$ contains at least one element of $B$, so the Hider wins with at least the probability that a random $w_{i}$ exceeds 1 , which is $V R^{c}(u, 1, h)=V R(u, u-1, h)$.

To prove the right hand inequality, set $u=C o-\operatorname{Ind}(\mathcal{S})$, and fix some maximal independent family $\mathcal{T}=\left\{S_{1}, \ldots, S_{u}\right\}$ of $\mathcal{S}$. Suppose the Searcher picks equiprobably from these. Then for any Hider weighting $w^{\prime}$ in $A^{c}(\mathcal{S})$, the Hider wins in $A^{c}(\mathcal{S})$ with the same probability that he wins in $R^{c}(u, 1, h)$ with weighting $\left(w^{\prime}\left(S_{1}\right), \ldots, w^{\prime}\left(S_{u}\right)\right)$, so in particular with probability no more that $V R^{c}(u, u-1, h)$.

For the table graph $C o-\operatorname{Ind}(\mathcal{S})=C o-\operatorname{Cov}(\mathcal{S})=2$ since one leg and the table top form a maximal independent family and one the node of the table top and the center node form a minimal covering set. The complementary accumulation game on the table graph is Ruckle's game with $n=2$ and $r=1$. Note that $C o-\operatorname{Ind}(\mathcal{S})+\operatorname{Cov}(\mathcal{S})=2+3=5$ equals the number of nodes of the table graph. This is true for general graphs by a classical theorem of Gallai [8]:

Theorem 21 If $\mathcal{S}$ is the family of edges of an ordinary graph then Co$\operatorname{Ind}(\mathcal{S})+\operatorname{Cov}(\mathcal{S})=|\mathcal{N}|$.

It is not hard to see that the complement of an independent node set of a graph is a node cover, which implies that $\operatorname{Ind}(\mathcal{S})+\operatorname{Co}-\operatorname{Cov}(\mathcal{S})=|\mathcal{N}|$ for graphs. In particular if $\operatorname{Ind}(\mathcal{S})=\operatorname{Cov}(\mathcal{S})$ then also $\operatorname{Co}-\operatorname{Ind}(\mathcal{S})=$ $\operatorname{Co}-\operatorname{Cov}(\mathcal{S})$. So the complementary accumulation game on a Ruckle graph is equivalent to Ruckle's complementary game.

\section{The Line and Circle Graphs}

In this section we study the complementary accumulation game on circle, with a brief preliminary look at the game on the infinite line. Since both are transitive, we seek to determine the maximum number $T$ of $s$-intervals (or $s$-arcs) $I$ that the Searcher can make heavy $(w(I) \geq 1)$ with total weight $h$. For the $n$-node circle graph, there are $n \quad s$-intervals, so the Value is simply $T / n$. For the infinite line we do not make a game interpretation but just 
consider the maximization problem for heavy intervals. In this section we will let $p, q$, and $e$ denote respectively the integer part, fractional part, and one minus the fractional part, of $h$.

$$
p=\lfloor h\rfloor, q=h-\lfloor h\rfloor=h-p \text {, and } e=\lceil h\rceil-h .
$$

\subsection{The infinite line graph $Z$}

On the integer graph $Z$, we show that the maximum number of $s$-intervals that the Hider can make heavy, given total weight $h$, is $p s=\lfloor h\rfloor s$. The proof of the following lemma is similar to a counting argument used by Katona [9] to give a short proof of the Erdos-Ko-Rado Theorem [7].

Lemma 22 Let $\mathcal{H}$ be a family of s-intervals of integers, with at most $p$ of them disjoint. Then the cardinality of $\mathcal{H}$ is no more than ps.

Proof. For $i=1, \ldots, s$, consider the family $\mathcal{F}_{i}$ of consecutive disjoint $s$ intervals $\{[k s+i, k s+i+s-1]\}_{k=-\infty}^{\infty}$, that is, their left endpoints are all $i(\bmod s)$. Since the sets in each family $\mathcal{F}_{i}$ are disjoint, we have $\mathcal{F}_{i} \cap \mathcal{H} \leq p$ and consequently $\left(\cup_{i=1}^{s} \mathcal{F}_{i}\right) \cap \mathcal{H} \leq p s$. But $\cup_{i=1}^{s} \mathcal{F}_{i}$ is the set of all $s$-intervals, so we are done.

Theorem 23 Let $w$ be a weighting on the integers with total weight $h$. Then the maximum number of heavy s-intervals of $w$ is no more than $p$ s. This number of heavy intervals is obtained by any weighting $w$ with weight 1 at $p$ integers spaced at distance at least s apart.

Proof. We have $h<\lfloor h\rfloor+1=p+1$. The total weight of any $p+1$ disjoint heavy sets would be at least $p+1$, which more than the total weight $h$. So the heavy sets of $w$ form a family of $s$-intervals with at most $p$ disjoint sets. By Lemma $22 w$ has no more than $p s$ heavy sets.

\subsection{Accumulation Games on the Circle}

On the circle graph, the accumulation game based on the search family of $r$-arcs is the same as the complementary accumulation game on $(s=(n-r))$ arcs. So it is enough to study the simpler complementary accumulation game on the circle graph $C_{n}$, where the Searcher picks an $s$-arc and the Hider wins if it is heavy. We denote the $s$-arcs by $A_{i}=\{i, i+1, \ldots, i+s-1\}$, assuming 
the nodes are numbered consecutively in say a clockwise manner around the circle. Since in this case the search set $\mathcal{S}$ of $s$-arcs is transitive, the problem (as noted in (4)) for the Hider is to choose the weighting $w$ which maximizes the number of heavy $s$-arcs. Note that the total number of $s$-arcs is $n$ (each one has it's anticlockwise endpoint at a different node of $\mathcal{N}$ ). We call this maximum number

$$
T=T(n, s, h),
$$

so the Value of the game is simply $T / n$. If $n \leq h s$, the Hider wins by placing weight $h / n$ at every node, so to avoid this trivial case we henceforth assume that $n>h s$.

This game was analyzed by Kikuta and Ruckle [12]. They obtained bounds on $T$ using the following observations. First, the Hider can always place $p$ weights of 1 with at least $s-1$ nodes between any two of them, which gives $s$ heavy $s$-arcs containing each 1 , or $p s$ in total. On the other hand, If we take the sum of $w\left(A_{i}\right)$ over all $s$-arcs for an optimal $w$, it must be at least $T \cdot 1=T$, and since this counts each node exactly $s$ times, the exact sum is $h s$. Hence the integer $T$ is bounded above by $\lfloor h s\rfloor$. In this way they obtained:

Theorem 24 (Kikuta-Ruckle) For all $n, s, h$, we have

$$
p s \leq T(n, s, h) \leq\lfloor h s\rfloor \text {. }
$$

We will improve both bounds where they are not tight.

Lemma 25 Let $w$ be a weighting on the nodes of $C_{n}$ for which some node $i$ is not in any heavy $s$-arc. Then $T \leq p s$.

Proof. Without loss of generality we may assume that $i=n$. Let $\hat{w}$ be the lift of $w$ to the integers, truncated to 0 outside the interval $\{1, \ldots, n-1\}$ that is, with $\hat{w}(i)=w(i)$ for $i=1, \ldots, n-1$, and $\hat{w}(i)=0$ otherwise. Note that the total weight $\hat{h}$ of $\hat{w}$ does not exceed $h$. Since $s<n$ by assumption, no heavy $s$-interval for $w$ can contain both 1 and $n-1$ (or else it would contain $n$ ) it follows that the heavy sets for $w$ and $\hat{w}$ are in one to one correspondence. Hence the result follows from Theorem 23.

Lemma 26 If $n>p(3 s-2)$ then there is a node $i$ in $C_{n}$ which is not in any heavy s-arc. 
Proof. As observed in the proof of Theorem 23, there are at most $p$ disjoint heavy $s$-arcs, which we may order clockwise as $H_{0}, \ldots, H_{p-1}$. Let $c_{k}$ denote the number of nodes clockwise of $H_{k}$ and anticlockwise of $H_{k+1}$, setting $H_{0}=H_{p}$. If every node $i$ belongs to some heavy $s$-arc, then in particular the midpoint(s) of the arcs between $H_{k}$ and $H_{k+1}$ does so. In particular, there is an $s$-arc containing the midpoint which intersects one of these two sets. Thus $\left|c_{k}\right| \leq 2(s-1)$ and so $n=p s+\sum_{k=0}^{p}\left|e_{k}\right| \leq p s+p 2(s-1)=p(3 s-2)$. The result follows from the contrapositive.

Combining both lemmas with the lower bound of Kikuta and Ruckle (7) gives the following solution of the circle game for large $n$.

Theorem 27 If $n>p(3 s-2)$, then $T=p s$.

For smaller values of $n$, we can also improve on the upper bound of (7) using a slightly modified counting argument.

If $s$ divides $n$, then set $k=n / s$ and for each node $i$, let $\mathcal{F}_{i}$ be the partition of the circle into $k$ consecutive $s$-arcs $A_{i, j}, j=1, \ldots, k$, with the first of these having anticlockwise endpoint of node $i$. For each $i$, at most $p$ of the $k$ arcs $A_{i, j}$ can be heavy, so at most $n p$ altogether. But each $s$-arc is counted $k$ times, so $T \leq n p / k=p s$, with equality holding from the lower bound of $(7)$.

Suppose now that $n=k s-e$, for some positive integer $e<s$. Let $\mathcal{F}_{i}$ be as before, but in this situation the $k s$-arcs cover the circle and are pairwise disjoint except for $A_{i, 1}$ and $A_{i, k}$, which overlap in an $e$-arc. Note that for each $i$,

$$
h=w(\mathcal{N})=\left(\sum_{j=1}^{k} w\left(A_{i, j}\right)\right)-w\left(K_{i}\right),
$$

where $K_{i}$ is the $e$-arc given by the overlap $A_{i, k} \cap A_{i, 1}$. Note that the only way that $p+1$ of the $\operatorname{arcs}$ in $\mathcal{F}_{i}$ can be heavy is if the $e$-arc $K_{i}$ is $(p+1-h)$-heavy, because in that case we must have from (8) that

$$
\begin{aligned}
w\left(K_{i}\right) & =\left(\sum_{j=1}^{k} w\left(A_{i, j}\right)\right)-h \\
& \geq(p+1)-h .
\end{aligned}
$$

Let $M$ denote the maximum number of $e$-arcs which can be $(p+1-h)$ - heavy. Note that by scaling the weights we have

$$
M=T(n, e, h /(p+1-h)) .
$$


So $p+1$ of the arcs in $\mathcal{F}_{i}$ can can be heavy for $M$ values of $i$, and only $p$ of them can be heavy for the remaining $n-M$ values of $i$, and hence the maximum number of heavy sets $A_{i, j}$ is

$$
M(p+1)+(n-M)(p)=M+p n .
$$

As above in the case where $s$ divides $n$, each $s$-arc is counted $k$ times, so we have proved (recall $p$ and $e$ defined in (6)) the following.

Theorem 28 For any $n, s, h$, we have for $k=\lceil n / s\rceil$ that,

$$
T=T(n, s, h) \leq\left\lfloor\frac{T(n, e, h /(1-(h-p)))+p n}{k}\right\rfloor .
$$

Example 29 Consider the circle game determined by $n=8, s=5$ and $h=6 / 5$ (with $p=1, k=2$ and $e=2$ ). The Kikuta-Ruckle upper bound ( 7$)$ is $T \leq\lfloor s h\rfloor=6$. Theorem 28 gives $T \leq(T(8,2, h /(1-(h-\lfloor h\rfloor)))+8) / 2=$ $(T(8,2,3 / 2)+8) / 2$. Since $n=8>\lfloor 3 / 2\rfloor(3 \cdot 2-2)=4$, it follows from Theorem 27 that $T(8,2,3 / 2)=\lfloor 3 / 2\rfloor 2=2$, and hence $T \leq(2+8) / 2=5$. This is tight, as the lower bound of Kikuta and Ruckle ( 7 ) gives $T \geq p s=5$.

The reason the example worked was that the overlap $e$ was small enough with respect to $n$ in order to apply Theorem 27. We can use this observation to generalize the example as follows.

Theorem 30 Given $n, s$ and $h$, define $p, k$ and $e$ as in Theorem 28.

$$
\text { If } e \leq \frac{s+2}{3} \text { then } T=T(n, s, h) \leq\left\lfloor\frac{e\lfloor h /(1-(h-p))\rfloor+p n}{k}\right\rfloor \text {. }
$$

Furthermore, if also the fractional part of $h$ is small in that $\lfloor h /(1-(h-\lfloor h\rfloor))\rfloor=$ $\lfloor h\rfloor$ (for example if $h$ is an integer), then

$$
T(n, s, h)=p s
$$

Proof. Since we are always assuming that $n>p s$, our assumption on $e$ gives

$$
n>p s>3 e-2 \text {. }
$$

It now follows from Theorem 27 that

$$
T(n, e, h /(1-(h-p))) \leq e\lfloor h /(1-(h-p))\rfloor .
$$


Then it follows from Theorem 28 that

$$
\begin{aligned}
T(n, s, h) & \leq\left\lfloor\frac{e\lfloor h /(1-(h-p))\rfloor+p n}{k}\right\rfloor, \text { which can be written } \\
& =\left\lfloor\frac{(\lceil n / s\rceil s-n)\lfloor h /(1-(h-\lfloor h\rfloor))\rfloor+\lfloor h\rfloor n}{\lceil n / s\rceil}\right\rfloor .
\end{aligned}
$$

If also

$$
\lfloor h /(1-(h-\lfloor h\rfloor))\rfloor=\lfloor h\rfloor
$$

then

$$
\begin{aligned}
T(n, s, h) & \leq\left\lfloor\frac{e p+p n}{k}\right\rfloor=\left\lfloor\frac{p(e+n)}{k}\right\rfloor \\
& =\left\lfloor\frac{p k s}{k}\right\rfloor=p s .
\end{aligned}
$$

But since $p s$ is a lower bound for $T$, we have in this case that

$$
T(n, s, h)=p s=\lfloor h\rfloor s, \text { as claimed. }
$$

The above result (the first part) gives a good bound on $T$, one close to $p s$ when $e$ is small. That is, when $n \bmod s$ is large, close to $s$. To obtain good upper bounds on $T$ when $q=n \bmod s$ is small (in particular, with $q$ equal to 0 or 1 ), we use the following argument. Suppose $n=k s+q$, $q<s$. By renumbering, we may assume that $A_{1}$ is a light set, where $A_{i}=$ $[i, i+s-1]$. For $j=1, \ldots, s$, let $F_{j}$ denote the family $k$ of consecutive $s$-arcs $A_{q+i}, A_{q+i+s}, \ldots, A_{q+i+k s}$, whose union is $\mathcal{N}-\{i, \ldots, i+q-1\}$. The $n=k s+q s$-arcs are comprised of the $k s s$-arcs in the $s$ families $F_{j}$, together with the $q s$-arcs $A_{1}, A_{2}, \ldots, A_{q}$. Since the arcs in each $F_{j}$ are disjoint, at most $p$ of them can be heavy. Since by assumption $A_{1}$ is light, at most $q-1$ of the $A_{i}$ can be heavy. Thus there can be at most $p s+q-1$ heavy sets, and we have proved the following.

Theorem 31 Suppose $n=k s+q, 1 \leq q<s$. Then

$$
T=T(n, s, h) \leq p s+q-1=\lfloor h\rfloor s+q-1 .
$$

Hence if $n \bmod s$ is 0 or 1 , then $T=p s$. In particular, this holds if $s \leq 2$. 
Note that if $q$ is large, we can get a better estimate from Theorem 30 using $e=s-q$, which will be small, and we will have $T=p s$. Note that the estimate (10) is in terms of $p=\lfloor h\rfloor$ and not of $h$. So it is likely to be good only when $h$ is close to $p+1$ (has a large fractional part).

Example 32 Let $n=14, s=6$ and $h=3 / 2$; thus $p=1$ and $q=2$. The estimate of Theorem 31 gives $T \leq 6+2-1=7$, while the upper bound of Kikuta and Ruckle (7) gives $T \leq\lfloor h s\rfloor=\lfloor(3 / 2) 6\rfloor=9$.

We can generalize this example to give a class of weightings which establish a lower bound that is sometimes useful. Suppose that $h=K / 2$ and $n \leq K(s-1)$ so that we can place $1 / 2$ 's at certain nodes to create heavy arcs which go around the circle (every node is in a heavy arc). Write $n=a K+b$, for positive integers $a$ and $b$ with $b<K$, so $a=\lfloor n / K\rfloor$. Then define a weighting $w$ which puts weight $1 / 2$ at $K$ nodes $x_{i}, i=1, \ldots, K$ with distances $d_{i}$ between $x_{i}$ and $x_{i+1}\left(\right.$ where $\left.x_{n+1}=x_{1}\right)$. Arrange it so $d_{i}=a+1$ for $b$ values of $i$ and $d_{i}=a$ for the remaining $K-b$ values. In general, the number of heavy $s$-arcs containing $x_{i}$ and $x_{i+1}$ is equal to $s-d_{i}$. Thus the total number of heavy $s$-arcs for this $w$ is given by

$$
b(s-(a+1))+(K-b)(s-a)=K(s-a)-b
$$

In the example we have $n=8, s=5, K=3$, so $a=\lfloor 8 / 3\rfloor=2$ and $b=8-2 \cdot 3=2$. Consequently the number of heavy 5 -arcs is $K(s-a)-b=$ $3(5-2)-2=7$. Setting $K=\lfloor 2 h\rfloor$

Theorem 33 Suppose that for some integer $K$ we have $h=K / 2$ and $n \leq$ $K(s-1)$. Then

$$
T \geq K(s-\lfloor n / K\rfloor)-(n-\lfloor n / K\rfloor) .
$$

More generally, if $n \leq\lfloor 2 h\rfloor(s-1)$ we have

$$
T \geq\lfloor 2 h\rfloor(s-\lfloor n /\lfloor 2 h\rfloor\rfloor)-(n-\lfloor n /\lfloor 2 h\rfloor\rfloor) .
$$

Similar bounds can be obtained when $h=K / a$, for integers $a<s$, by using weights of $1 / a$. 


\section{Conclusions}

In their original paper on accumulation games on discrete locations, Kikuta and Ruckle briefly considered some extensions to case where the hiding locations had the structure of a graph; namely the circle and interval graphs. Motivated by those examples, we have extended the general definition of an accumulation game to allow the Searcher to search any set $S$ belonging to a given family (hypergraph) of subsets of hiding locations. Unlike the original games of Kikuta and Ruckle, which were equivalent to optimization problems for the Hider, our extension is a true game where both players must act strategically, and in general both need to adopt mixed strategies.

We conclude with an observation on a connection with reliability theory. Suppose there are $n$ power plants connected in some network structure. Each day a weight $w_{i}$ of coal must be delivered to plant $i$, subject to a bound on total coal. Due to various potential faults in the network structure, there is a small probability that certain sets $S$ of plants will simultaneously fail (e.g. those which share a common link to the main network). The probability that more than one such set $S$ fails is negligible. How should the coal be allocated to maximize the probability that some minimum power is generated? If the collection of sets of plants that might simultaneously fail is denoted $\mathcal{S}$, this is a form of the accumulation game $A(\mathcal{S})$. 


\section{References}

[1] S. Alpern and M. Asic (1985), The search value of a network. Networks, $15,229-238$.

[2] S. Alpern and S. Gal (1988), A Mixed Strategy Minimax Theorem without Compactness, SIAM J. Control and Optimization, Vol. 26, 13571361.

[3] S. Alpern and S. Gal (2003). The Theory of Search Games and Rendezvous. Kluwer International Series in Operations Research and Management Sciences, 319 pp, Kluwer, Boston.

[4] V. J. Baston, F. A. Bostock and T. S. Ferguson (1989), The number hides game, Proceedings of the American Mathematical Society, Vol. 107, No. 2 , pp. 437-447

[5] B. Bollobas (1986), Combinatorics, Cambridge University Press, Cambridge, 1986.

[6] J. Dally, N. Clayton, N. Emery (2006), The behaviour and evolution of cache protection and pilferage, Animal Behaviour 72, Issue 1, 13-23.

[7] P. Erdös, C. Ko, R. Rado (1961), Intersection theorems for systems of finite sets, Quart. J. Math. Oxford Ser. (2) 12 , 313-320.

[8] T. Gallai (1959), Uber extreme Punkt- und Kantenmengen, Ann. Univ.Sci.Eötvös Sect. Math. 2, 133-138.

[9] G. O. H. Katona (1972). A simple proof of the Erdös-Chao Ko-Rado theorem. Journal of Combinatorial Theory, Series B, 13, 183-184.

[10] K. Kikuta, W. Ruckle (1997), Accumulation games, Part 1: noisy search, J. Optimiz. Th. Appl. 94, no. 2, 395-408.

[11] K. Kikuta, W. Ruckle (2000), Continuous accumulation games in continuous regions, J. Optimiz. Th. Appl. 106, no. 3, 581-601.

[12] K. Kikuta, W. Ruckle (2001), Continuous accumulation games on discrete locations, Naval Res. Logist. 41, no. 6, 821-831. 
[13] D. Kőnig (1931). Gráfok és mátrixok. Matematikai és Fizikai Lapok 38, 116-119.

[14] D. Morris (1962). The behaviour of the green acouchi (Myoprocta pratti) with special reference to scatter hoarding. Proc Zool Soc Lond 139, 701732 .

[15] W. H. Ruckle (1983), Geometric Games and Their Applications, Pitman Press, Boston Massachusetts.

[16] W. Ruckle, Accumulation games, Sci. Math. Jpn. 54 (2001), no. 1, 173203.

[17] Z. Tuza (1991), Extensions of Gallai's graph covering theorems for Uniform Hypergraphs, J. Combin. Th. (B) 52, 92-96.

[18] S. B. Vander Wall, J. S. Briggs, S. H. Jenkins, K. M. Kuhn, T. C. Thayer and M. J. Beck (2006), Do food-hoarding animals have a cache recovery advantage? Determining recovery of stored food, Animal Behaviour 72, no. 1, 189-197.

[19] N. Zoroa, M. J. Fernandez-Saez, P. Zoroa (1999), A game related to the number of hides game, J. Optimiz. Th. Appl. 103, No. 2, pp. 457-473. 